Agron. Mesoam. 29(2):251-261. Mayo-agosto, 2018

ISSN 2215-3608, doi:10.15517/ma.v29i2.29900

http://www.revistas.ucr.ac.cr/index.php/agromeso

\title{
Caracterización estomática, concentración de clorofila y su relación con producción de biomasa en Bouteloua curtipendula ${ }^{1}$
}

\section{Stomatal characterization, chlorophyll concentration and their relation with biomass production of Bouteloua curtipendula}

\author{
Alan Álvarez-Holguín ${ }^{2}$,Carlos Raúl Morales-Nieto², Raúl Corrales-Lerma², Carlos Hugo Avendaño-Arrazate ${ }^{3}$, \\ Héctor Oswaldo Rubio-Arias ${ }^{2}$,Federico Villarreal-Guerrero ${ }^{2}$
}

\begin{abstract}
Resumen
Las características de los estomas y la concentración de clorofila son de los principales factores que determinan la productividad de una planta. El objetivo del presente estudio fue caracterizar la distribución y densidad de estomas, estimar la concentración de clorofila y evaluar su efecto en la producción de biomasa en genotipos de "pasto banderita" [Bouteloua curtipendula (Michx.) Torr.]. El experimento se realizó de mayo a julio del 2015, bajo condiciones de invernadero en la Facultad de Zootecnia y Ecología (FZyE) de la Universidad Autónoma de Chihuahua (UACH), México. Se evaluaron tres variedades comerciales: El Reno, Niner y Vaughn, y dos genotipos nativos: E-689 y E-592. Las variables evaluadas fueron índice de concentración de clorofila (ICC), densidad estomática (DE), densidad de tricomas (DT), índice estomático (IE) y área estomática (AE). Estas se relacionaron mediante análisis de regresión con producción de biomasa (PB). La DE y el IE tuvieron relación negativa con la PB, mientras que la AE y el ICC se relacionaron de forma positiva. El genotipo E-689 presentó la menor DE e IE $(p<0,05)$, los valores fluctuaron desde 152,7 hasta 275 estomas $/ \mu \mathrm{m}^{2}$ y de 13,41 a $16,03 \%$, respectivamente. Además, presentó mayor AE y PB (p<0,05), fluctuando de 186,7 a $361,7 \mu \mathrm{m}^{2}$ y de 13,5 a 30,3 g. Los genotipos de pasto banderita con baja densidad estomática e índice estomático y alta área estomática e índice de concentración de clorofila, pueden producir mayor cantidad de biomasa.
\end{abstract}

Palabras clave: estoma, tricomas, rendimiento de cultivos, forrajes.

\begin{abstract}
The stomatal characteristics and chlorophyll concentration are some of the main parameters, to determine plant productivity. The objective of the present study was to characterize the stomatal density and distribution to estimate the chlorophyll concentration and evaluate their effect on the biomass production of sideoats grama [Bouteloua curtipendula (Michx.) Torr.] genotypes. The experiment was performed from May to July 2015 under greenhouse
\end{abstract}

\footnotetext{
Recibido: 27 de julio, 2017. Aceptado: 4 de octubre, 2017. Este trabajo formó parte de la tesis de maestría del primer autor realizada en la Facultad de Zootecnia y Ecología, Universidad Autónoma de Chihuahua. México.

2 Universidad Autónoma de Chihuahua, Facultad de Zootecnia y Ecología. Periférico Francisco R. Almada kilómetro 1. Código postal 33820. Chihuahua, México. alvarezalan193@gmail.com, cnieto@uach.mx (autor para correspondencia),rulo.crr.ler@gmail.com, rubioa1105@ hotmail.com, toloc604@email.arizona.edu

3 Instituto Nacional de Investigaciones Forestales, Agrícolas y Pecuarias (INIFAP), Campo experimental Rosario Izapa. Carretera Tapachula Cacahoatán kilómetro 18. Código postal 30870. Tuxtla Chico, Chiapas, México. avendano.carlos@inifap.gob.mx
} 
conditions, at- the School of Animal Sciences and Ecology of the Autonomous University of Chihuahua, Mexico. Three commercial varieties were studied: El Reno, Niner, Vaughn and two native genotypes: E-689 and E-592 of sideoats grama. The variables evaluated were chlorophyll concentration index (CCI), stomatal density (SD), trichome density (TD), stomatal index (SI), and stomatal area (SA). These variables were related to biomass production (BP) applying regression analysis. SD and SI showed a negative effect on BP, while SA and CCI showed a positive effect. The genotype E-689 showed the lowest $(\mathrm{p}<0.05) \mathrm{SD}$ and SI, with values from 152.7 to $275 \mathrm{stomatal} / \mu \mathrm{m}^{2}$ and from 13.41 to $16.03 \%$, respectively. In addition, it also presented the highest $(\mathrm{p}<0.05) \mathrm{SA}$ and $\mathrm{BP}$, with values from 186.7 to $361.7 \mu \mathrm{m}^{2}$ and 13.5 to $30.3 \mathrm{~g}$, respectively. In conclusion, genotypes of sideoats grama with lower stomataldensity and stomatal index and higher stomatal area and chlorophyll concentration index, could produce higher amounts of biomass.

Keywords: stomata, trichomes, crop yield, forage.

\section{Introducción}

La mayor parte de los pastizales en zonas áridas y semi áridas de México se encuentran degradados, principalmente por causa del sobrepastoreo (Melgoza-Castillo et al., 2007). Para atender estos ecosistemas degradados existen diversas técnicas y metodologías, dentro de las cuales se considera a la resiembra de pastos. Sin embargo, una desventaja económica de una resiembra es que, alrededor del $90 \%$ de la semilla que se utiliza se importa (Palma-Rivero et al., 2000).

El "pasto banderita" [Bouteloua curtipendula (Michx.) Torr.] de la familia Poaceae, es una de las especies más utilizada en la resiembra de pastizales en el norte de México, ya que existe gran variabilidad genética de esta especie en el país (Morales-Nieto et al., 2006; 2008). A pesar de la importancia de este pasto con potencial de utilización en más de setenta millones de hectáreas en México, solo se ha generado una variedad (Beltrán et al., 2013). Por esta razón, es prioritaria la selección de ecotipos nativos que puedan convertirse en variedades adaptadas a las condiciones locales. Es importante que, al seleccionar ecotipos se considere su aparato estomático y concentración de clorofila, debido a que, el tamaño y densidad de estomas son factores de importancia en la utilización del agua y en el dinamismo de la fotosíntesis y, por lo tanto, en la productividad de las plantas (Lawson et al., 2014). La clorofila es un pigmento capaz de captar fotones de luz solar de diferentes colores y convertirlos en compuestos orgánicos ricos en energía, por el proceso de fotosíntesis (Ghasemi et al., 2011). Debido a esto, la concentración de clorofila representa una medida directa de la eficiencia del aparato asimilador de radiación solar y se correlaciona con la producción de materia seca (Rivera et al., 2005; Rodrigues et al., 2006; Wu et al., 2008). El objetivo del presente estudio fue caracterizar la distribución y densidad de estomas, estimar la concentración de clorofila y evaluar su efecto en la producción de biomasa en genotipos de "pasto banderita".

\section{Materiales y métodos}

El experimento se realizó de mayo a julio del 2015, en condiciones de invernadero en la Facultad de Zootecnia y Ecología (FZyE) de la Universidad Autónoma de Chihuahua (UACH), México. Se evaluaron las variedades comerciales El Reno, Niner y Vaughn, procedentes de Estados Unidos de Norteamérica; así como, dos ecotipos nacionales, E-689 y E-592, seleccionados como sobresalientes de un proceso de evaluación de 135 ecotipos recolectados en distintas ecorregiones del norte de México (Morales et al., 2016). El genotipo E-689 fue recolectado en un pastizal amacollado, en el tramo El Sauz-Namiquipa en el estado de Chihuahua, mientras que, el genotipo E-592 se recolectó en la carretera de cuota Ojinaga-Chihuahua, en un matorral dominado por Larrea 
tridentata (DC.) Coville.

En bolsas de polietileno negro de $26 \mathrm{~cm}$ de altura por $18 \mathrm{~cm}$ de diámetro, se sembró semilla producida por los ecotipos sobresalientes y las variedades comerciales, y se dejó una planta por bolsa. Para facilitar la posterior extracción de las raíces, se utilizó arena como sustrato. Al agua de riego se le adicionaron a razón de 2 g/l de fertilizante triple 18 (18N-18P-18K), con el propósito de compensar la falta de nutrientes en la arena. Los riegos se realizaron cada dos o tres días hasta su punto de saturación, según el desecamiento del sustrato. Durante el experimento, en el invernadero se monitoreó la temperatura (T) y humedad relativa (HR) con la sonda modelo HMP60. Los datos se registraron en un datalogger CR1000. La T media durante el experimento fue de 26,7 \pm $5,6^{\circ} \mathrm{C}$, con mínima de $17,1^{\circ} \mathrm{C}$ y máxima de $44,7^{\circ} \mathrm{C}$, mientras que, la $\mathrm{HR}$ media fue de $52,0 \pm 16,8 \%$.

El experimento se llevó a cabo bajo un diseño completamente al azar. Se evaluó semanalmente la producción de biomasa (PB) durante setenta días, a partir de la emergencia de plántulas. Para ello, se pesaron en seco cinco plantas completas (raíz y parte aérea) por genotipo, en cada uno de los diez muestreos realizados. La raíz se retiró de la arena lavándola con agua, y el secado de planta se realizó en una estufa a $65^{\circ} \mathrm{C}$ durante $72 \mathrm{~h}$. El pesaje se hizo en una balanza analítica con precisión de $0,01 \mathrm{~g}$. Otra variable evaluada fue el índice de concentración de clorofila, el cual se calculó con el dispositivo CCM-200, semanalmente durante setenta días. Las mediciones se realizaron en la parte media de diez hojas maduras y sanas, seleccionadas al azar por planta, y se analizaron cinco plantas por genotipo.

Se evaluó la densidad estomática (DE), densidad de tricomas (DT) e índice estomático (IE) por milímetro cuadrado $\left(\mathrm{mm}^{2}\right)$, y el área estomática (AE) en micrómetros cuadrados $\left(\mu \mathrm{m}^{2}\right)$. Esto se realizó en una muestra de cinco plantas de diez semanas de edad en cada genotipo, en las que, se seleccionaron tres hojas maduras de cada planta. Se obtuvo la impresión aplicando una capa de barniz o esmalte transparente de uñas en un segmento de aproximadamente $1 \mathrm{~cm}$, en el sentido de las nervaduras. Este procedimiento se realizó en las superficies adaxial y abaxial, se dejó secar el barniz por 20 min hasta que se solidificó y se retiró con pinzas de disección, con cuidado de no estirar la placa de esmalte. Las muestras se colocaron sobre un porta-objetos y se cubrieron con un cubreobjetos; luego se observaron a campo claro en un microscopio óptico con un aumento de 400 diámetros (400x), y se tomaron fotografías de cinco campos ópticos de $0,0945 \mathrm{~mm}^{2}$, seleccionados al azar con una cámara, posteriormente se traspoló a $1 \mathrm{~mm}^{2}$. En cada campo óptico se contabilizó el número total de células epidérmicas (NCE), estomas y tricomas, para luego determinar la densidad por milímetro cuadrado $\left(\mathrm{mm}^{2}\right)$, la DE y la DT. El área estomática (AE) se calculó con la información de tres estomas seleccionados al azar, mediante el software Zen 2 core. Con el NCE y el número de estomas (NE) se calculó el índice estomático (IE), mediante la ecuación de Salisbury (1927) indicada por Wilkinson (1979): \% IE = [NE/(NCE+NE) $] 100$. Las variables DE, IE, DT y AE se calcularon por separado para las superficies adaxial y abaxial.

Para describir la relación de PB con relación al ICC a través de las semanas, se realizó un análisis de regresión. Los valores de PB de la décima semana se analizaron mediante regresión, con los datos de las variables DE, IE y AE de las zonas adaxial y abaxial por separado. Las variables DE, IE, DT y AE se analizaron con análisis de varianza (ANOVA). Para conocer diferencias entre medias se utilizó la prueba de Tukey, comparando la parte abaxial y adaxial por separado entre genotipos, así como, la parte abaxial y adaxial en general. Todos los análisis se realizaron con el procedimiento GLM del paquete estadístico SAS (2006), a un nivel de significancia de $0,05(\alpha=0,05)$.

\section{Resultados}

De acuerdo con el análisis estadístico para la densidad estomática (DE) de las superficies abaxial (DEE) y adaxial $(\mathrm{DEH})$, se encontraron diferencias $(\mathrm{p}<0,0001)$ entre genotipos. Los genotipos con mayor DEE y DEH $(\mathrm{p}<0,05)$ fueron las variedades comerciales, mientras que, los de menor fueron los genotipos nativos (Figura 1). Respecto al índice estomático (IE), tanto en la zona abaxial (IEE) como en la adaxial (IEH), solo E-689 mostró 


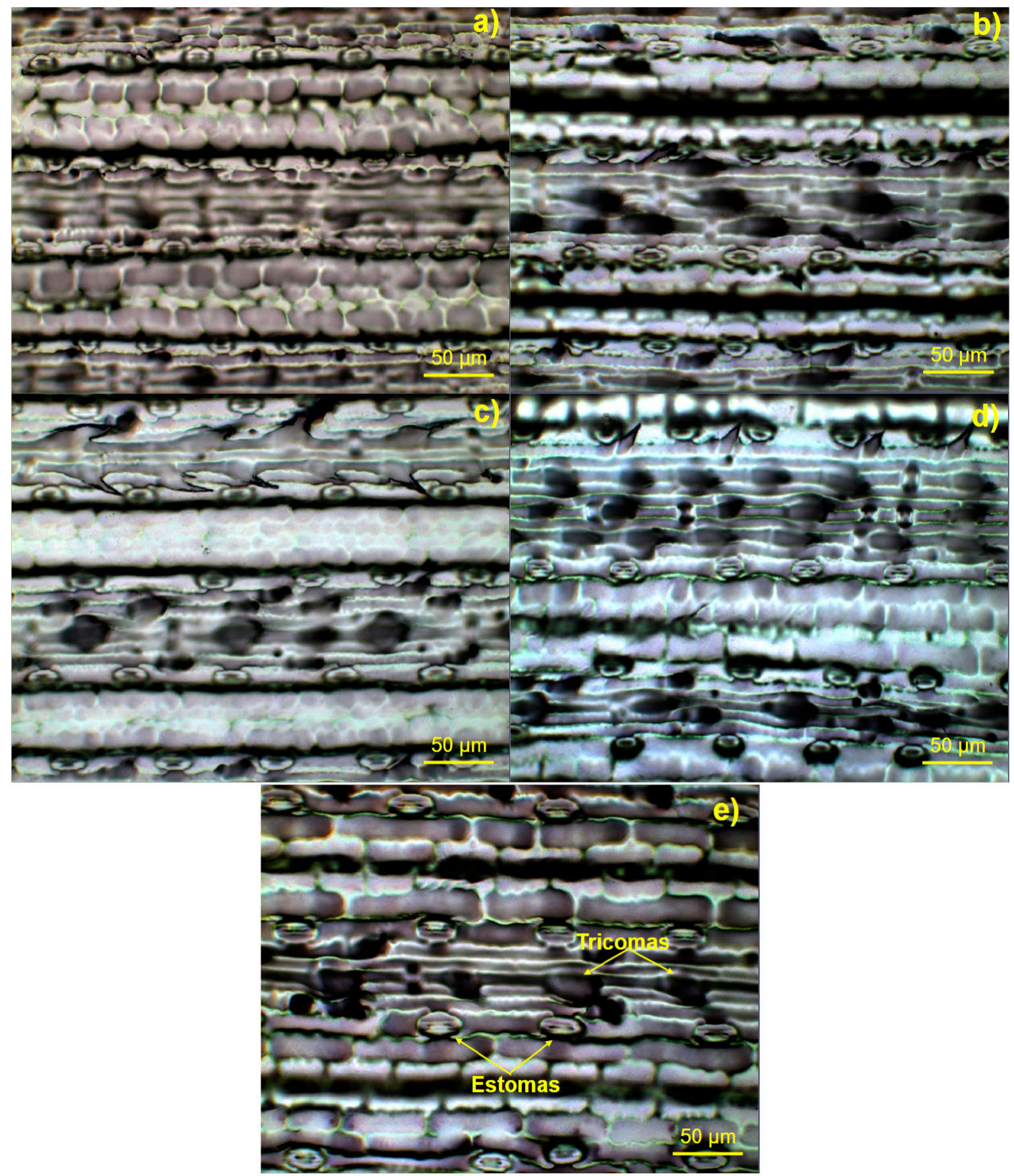

Figura 1. Micromorfología foliar en condiciones de invernadero de cinco genotipos de pasto banderita (Bouteloua curtipendula), con potencial para su uso en revegetación de pastizales. Facultad de Zootecnia y Ecología (FZyE), Universidad Autónoma de Chihuahua (UACH), México. 2015.

$\mathrm{a}=$ El Reno, $\mathrm{b}=$ Vaughn, $\mathrm{c}=$ Niner, $\mathrm{d}=\mathrm{E}-592$, e= E-689.

Figure 1. Leaf micromorphology under greenhouse conditions of five sideoats grama (Bouteloua curtipendula) genotypes, used in grasslands revegetation. School of Animal Sciences and Ecology, Autonomous University of Chihuahua (UACH), Mexico. 2015.

$\mathrm{a}=$ El Reno, $\mathrm{b}=$ Vaughn, $\mathrm{c}=$ Niner, $\mathrm{d}=\mathrm{E}-592$, e= E-689. 
diferencias significativas $(\mathrm{p}<0,05)$, al presentar los valores más bajos. Niner y E-592 presentaron mayor $(\mathrm{p}<0,05)$ densidad de tricomas (DT) en el envés (DTE), seguidos por Vaughn y El Reno, mientras que, E-689 presentó la menor (p<0,05) DTE. En la DT en el haz (DTH), E-689 fue el único que presentó diferencias, con la menor DTH, y mostró la mayor $(\mathrm{p}<0,05)$ área estomática $(\mathrm{AE})$, tanto del área abaxial $(\mathrm{AEE})$ como la adaxial $(\mathrm{AEH})$, seguido por E-592. Los genotipos con mayor AEE y AEH fueron Niner y El Reno, mientras que, Vaughn presentó la menor AEE y AEH.

En general, las variables DE y AE no presentaron diferencias $(\mathrm{p}<0,05)$ entre las superficies adaxial y abaxial, pero si para IE y DT $(\mathrm{p}<0,05)$ (Cuadro 1$)$. El comportamiento de la DE mostró una relación cuadrática $(\mathrm{p}<0,05)$ negativa con la producción de biomasa (PB), y no se presentaron diferencias $(\mathrm{p}>0,05)$ entre ambas superficies de la hoja. Con relación a este resultado, el genotipo con menor DE fue E-689, el cual presentó una mayor PB, mientras que, el de menor DE fue El Reno, que presentó una menor PB (Figura 2a). El IE y la PB mostraron una relación cuadrática $(p<0,05)$ inversa, y se presentaron diferencias $(p<0,05)$ entre las dos superficies. El genotipo con menor IE fue E-689, tanto en en la superficie adaxial como abaxial y una mayor PB, en tanto que, los genotipos con mayor IE presentaron una menor PB ( $\mathrm{p}<0,05$; Figura $2 b)$.

La variable AE mostró una relación cuadrática $(\mathrm{p}<0,05)$ positiva con la $\mathrm{PB}$, y no se presentaron diferencias $(\mathrm{p}<0,05)$ entre las dos superficies de la hoja (Figura 2c). Para esta variable, el genotipo con una mayor AE ( $<<0,05)$, tanto en superficie adaxial como abaxial fue E-689, mientras que, Vaughn presentó la menor $\mathrm{AE}(\mathrm{p}<0,05)$ en ambos lados. Niner y E-592 presentaron mayor DT $(\mathrm{p}<0,05)$ en ambas superficies, por su parte, E-689 presentó los valores más bajos $(\mathrm{p}<0,05)$. En esta variable se presentaron diferencias $(\mathrm{p}<0,05)$ entre las dos superficies, donde la mayor DT se presentó en la superficie abaxial (Cuadro 1).

En la Figura 3 se muestra el comportamiento de la PB conforme al índice de concentración de clorofila (ICC) a través de las semanas, con una tendencia cuadrática $(\mathrm{P}<0,001)$ en todos los genotipos. Además, se presentaron diferencias $(p<0,001)$ entre genotipos, y los coeficientes de determinación $\left(R^{2}\right)$ fluctuaron en un rango desde 0,64 hasta 0,96. El único genotipo con diferencias marcadas en el ICC fue E-689, el cual presentó los mayores valores de $\mathrm{PB}$, mientras que, los demás genotipos presentaron comportamiento similar en el aumento de ICC. Por lo anterior, el ICC parece tener un efecto sobre las diferencias entre los genotipos en PB.

\section{Discusión}

La densidad de estomas es un parámetro importante en la ecofisiología de las plantas, ya que controlan la pérdida de agua por transpiración y la ingesta de $\mathrm{CO}_{2}$ (Lawson y Blatt, 2014; Lawson et al., 2014). Está documentado que plantas con mayor densidad de estomas (DE), presentan menor tasa de transpiración (Schlüter et al., 2003; Franks y Beerling, 2009), lo cual puede tener relevancia en condiciones de aridez, ya que en estos ecosistemas las tasas de evapotranspiración son más altas. Lo anterior, posiblemente se debe a que, con DE bajas, la resistencia estomática aumenta y, en consecuencia, se limita el exceso de transpiración (Lawson y Blatt, 2014). Esta afirmación concuerda con lo mencionado por Xu y Zhou (2008), quienes encontraron que a mayor tamaño y menor densidad de estomas, la evapotranspiración disminuyó en el pasto Leymus chinensis (Trin.) Tzevelev. Por lo anterior, el número de estomas incluso ha sido foco de trabajos de manipulación genética, donde Franks et al. (2015), lograron incrementar la eficiencia en el uso del agua en Arabidopsis, al inducir una disminución en la DE. En general, los genotipos nativos presentaron menor DE al compararlos con los genotipos comerciales, lo cual indica que posiblemente poseen mayor resistencia estomática y por lo tanto, pueden presentar mayor tolerancia al estrés hídrico.

La DE también está relacionada con la PB, al respecto Fraser et al. (2009), vincularon la densidad de estomas con la producción de biomasa en Pseudoroegneria spicata (Pursh) Á. Löve, y notaron que, individuos con menor densidad de estomas presentaron mayor producción. De igual manera, Meharun-Nisa et al. (2009) observaron 
Cuadro 1. Índice y caracterización estomática en condiciones de invernadero de cinco genotipos de pasto banderita (Bouteloua curtipendula), con potencial para su uso en la revegetación de pastizales. Facultad de Zootecnia y Ecología (FZyE), de la Universidad Autónoma de Chihuahua (UACH), México. 2015.

Table 1. Stomatal index and characterization of five sideoats grama (Bouteloua curtipendula) genotypes, used in grasslands revegetation. School of Animal Sciences and Ecology Autonomous University of Chihuahua (UACH), México. 2015.

\begin{tabular}{lcccccccc}
\hline Genotipo & DEE & DEH & IEE & IEH & DTE & DTH & AEE & AEH \\
\hline E-689 & $176,6 \pm 2,7^{\mathrm{e}}$ & $152,7 \pm 2,8^{\mathrm{c}}$ & $15,6 \pm 0,3^{\mathrm{b}}$ & $13,4 \pm 0,3^{\mathrm{c}}$ & $230,0 \pm 8,6^{\mathrm{d}}$ & $204,4 \pm 7,1^{\mathrm{b}}$ & $358,6 \pm 3,3^{\mathrm{a}}$ & $361,7 \pm 3,5^{\mathrm{a}}$ \\
E-592 & $199,9 \pm 4,2^{\mathrm{d}}$ & $187,3 \pm 5,0^{\mathrm{d}}$ & $17,4 \pm 0,2^{\mathrm{a}}$ & $14,2 \pm 0,3^{\mathrm{c}}$ & $346,6 \pm 13^{\mathrm{ba}}$ & $257,3 \pm 11,9^{\mathrm{a}}$ & $310,2 \pm 20,2^{\mathrm{b}}$ & $294,6 \pm 16,8^{\mathrm{b}}$ \\
Vaughn & $248,5 \pm 7,5^{\mathrm{b}}$ & $257,7 \pm 4,9^{\mathrm{b}}$ & $17,3 \pm 0,4^{\mathrm{a}}$ & $16,0 \pm 0,2^{\mathrm{a}}$ & $309,7 \pm 6,5^{\mathrm{bc}}$ & $285,0 \pm 8,3^{\mathrm{a}}$ & $186,7 \pm 2,4^{\mathrm{d}}$ & $192,9 \pm 2,3^{\mathrm{d}}$ \\
Niner & $217,8 \pm 3,8^{\mathrm{c}}$ & $208,9 \pm 3,8^{\mathrm{c}}$ & $17,3 \pm 0,3^{\mathrm{a}}$ & $15,2 \pm 0,3^{\mathrm{ab}}$ & $370,6 \pm 11,8^{\mathrm{a}}$ & $290,9 \pm 7,1^{\mathrm{a}}$ & $233,7 \pm 2,8^{\mathrm{c}}$ & $232,1 \pm 3,2^{\mathrm{c}}$ \\
El Reno & $275,0 \pm 8,7^{\mathrm{a}}$ & $273,1 \pm 5,0^{\mathrm{a}}$ & $17,7 \pm 0,4^{\mathrm{a}}$ & $15,6 \pm 0,3^{\mathrm{a}}$ & $302,5 \pm 9,7^{\mathrm{c}}$ & $270,7 \pm 12,6^{\mathrm{a}}$ & $221,8 \pm 4,5^{\mathrm{c}}$ & $204,5 \pm 5,2^{\mathrm{cd}}$ \\
Media general & $224,0 \pm 3,4^{\mathrm{a}}$ & $215,9 \pm 3,9^{\mathrm{a}}$ & $17,1 \pm 0,1^{\mathrm{a}}$ & $14,9 \pm 0,1^{\mathrm{b}}$ & $311,9 \pm 5,6^{\mathrm{a}}$ & $261,2 \pm 4,7^{\mathrm{b}}$ & $262,2 \pm 2,9^{\mathrm{a}}$ & $257,1 \pm 4,4^{\mathrm{a}}$ \\
\hline
\end{tabular}

* Medias con letras diferentes representan diferencias estadísticas significativas (Tukey; $\mathrm{p}<0,05)$. DEE $=$ densidad estomática de la superficie abaxial $\left(\mathrm{mm}^{2}\right)$. DEH= densidad estomática de la superficie adaxial $\left(\mathrm{mm}^{2}\right)$. IEE= índice estomático del área abaxial $\left(\mathrm{mm}^{2}\right)$. $\mathrm{IEH}=$ índice estomático del área adaxial $\left(\mathrm{mm}^{2}\right)$. DTE= densidad de tricomas de la zona abaxial $\left(\mathrm{mm}^{2}\right)$. DTH= densidad de tricomas de la superficie adaxial $\left(\mathrm{mm}^{2}\right)$. AEE $=$ área estomática de la superficie abaxial $\left(\mu \mathrm{m}^{2}\right)$. AEH= área estomática de la superficie adaxial $\left(\mu \mathrm{m}^{2}\right)$. Letras diferentes en la media general representan diferencias estadísticas (Tukey; $p<0,05$ ) entre ambas superficies, sin discriminar entre genotipos / Means with different letters are statistically different (Tukey; $\mathrm{p}<0,05$ ). DEE $=$ stomatal density on the abaxial leaf surface. $\mathrm{DEH}=$ stomatal density on the abaxial leaf surface $\left(\mu \mathrm{m}^{2}\right)$. IEE $=$ stomatal index on the abaxial leaf surface $\left(\mu \mathrm{m}^{2}\right)$. IEH $=$ stomatal index on the adaxial leaf surface $\left(\mu \mathrm{m}^{2}\right)$. DTE $=$ trichomes density on the underside of leaf $\left(\mu \mathrm{m}^{2}\right)$. DTH $=$ trichomes density on the upper side of leaf $\left(\mu \mathrm{m}^{2}\right) . \mathrm{AEE}=$ stomatal area on the abaxial leaf surface $\left(\mu \mathrm{m}^{2}\right) . \mathrm{AEH}=$ stomatal area on the adaxial leaf surface $\left(\mu \mathrm{m}^{2}\right)$. Different letters on the general mean represents statistical differences (Tukey; $\mathrm{p}<0,05)$ between the two surfaces, without discriminating among genotypes.

que, el rendimiento de trigo (Triticum aestivum L.) fue mayor en genotipos con menor DE. Esto concuerda con los resultados obtenidos, donde el genotipo E-689 al presentar la menor DE obtuvo mayor producción de biomasa $(\mathrm{PB})$, mientras que El Reno al presentar la mayor DE obtuvo menor PB.

Las especies con mayor proporción de estomas en la superficie adaxial son identificadas como epiestomáticas, mientras que, con una mayor proporción de estomas en la superficie abaxial se clasifican como hipoestomáticas. Respecto a las especies que poseen la misma proporción de estomas en ambas superficies se clasifican como anfiestomáticas (Perveen et al., 2007; Reyes-López et al., 2015). En general, en este estudio no se presentaron diferencias entre ambas superficies de la hoja; por tanto, se puede clasificar al pasto banderita como una especie anfiestomática. La presencia de estomas en ambos lados de la hoja contribuye con una mayor tasa fotosintética y, al mismo tiempo, aporta una mayor difusión de $\mathrm{CO}_{2}$ entre las células del mesófilo. En especies $\mathrm{C} 4$ como el pasto banderita, que no presentan el problema de la fotorespiración, esto permite que la planta continúe fotosintetizando aún con sus estomas cerrados. Esto incrementa la eficiencia en el uso del agua, ya que, reduce la pérdida por transpiración (Parkhurst et al., 1988; Klooster y Plamer-Young, 2004).

La densidad de estomas tiene efecto en la fotosíntesis y en la transpiración. Sin embargo, el número de estomas puede variar con relación a la parte de las hojas en que se calcula y la edad de las mismas (Martin y Estimart, 2005). Además, la DE tiene la desventaja de ser afectada por las condiciones ambientales (Walton, 1980). El índice estomático, definido como la relación entre el número de estomas y la cantidad de células epidérmicas, es una variable menos susceptible a los factores antes mencionados. Por esta razón, es frecuentemente utilizada para comparar el número de estomas por área entre genotipos (Croxdale, 2000; Wang et al., 2007). Al respecto, en este estudio el IE varió entre los genotipos y además, presentó una relación negativa con la PB; por lo que, el genotipo E-689 con menor IE fue el que presentó la mayor PB. Los tricomas son estructuras uni y pluricelulares presentes 

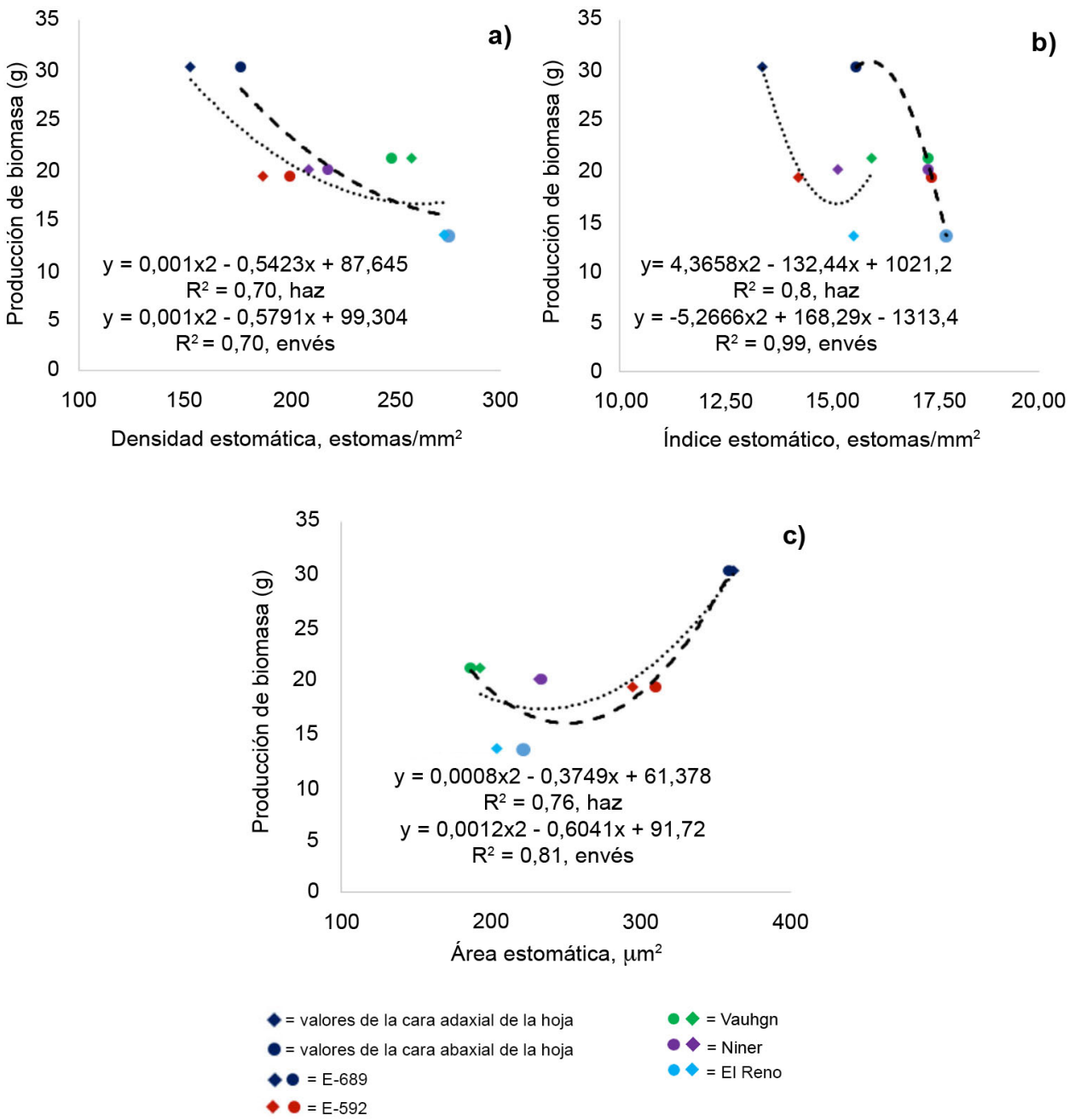

Figura 2. Relación entre la densidad estomática (a), índice estomático (b) y área estomática (c) con la producción de biomas en cinco genotipos de pasto banderita (Bouteloua curtipendula), con potencial para su uso en la revegetación de pastizales. Facultad de Zootecnia y Ecología (FZyE), Universidad Autónoma de Chihuahua (UACH), México. 2015.

Figure 2. Impact of stomatal density (a), stomatal index (b) and stomatal area (c) on biomass production on five sideoats grama (Bouteloua curtipendula) genotypes, used in grasslands revegetation. School of Animal Sciences and Ecology, Autonomous University of Chihuahua (UACH), México. 2015.

en diferentes partes de la epidermis de las plantas, cuya función principal es proteger a la planta de la radiación solar y del viento y como protección para reducir la pérdida por transpiración (Duke, 1994). Por esta razón, los genotipos con mayor DT (Niner, Vaugh y E-592), pueden tener ventaja adaptativa al compararlos con los de menor DT (E-689 y El Reno).

El área estomática indica el tamaño de las células guarda y del poro en conjunto. Los estomas de mayor tamaño generalmente poseen poros más grandes (Taylor et al., 2012; Giday et al., 2013). El área del poro y, por lo tanto, el AE se relacionan con mayor ingesta de $\mathrm{CO}_{2}$, lo que puede beneficiar el rendimiento en la especie. Lo anterior concuerda con los resultados obtenidos, donde el genotipo que presentó mayor AE (E-689), fue el que 


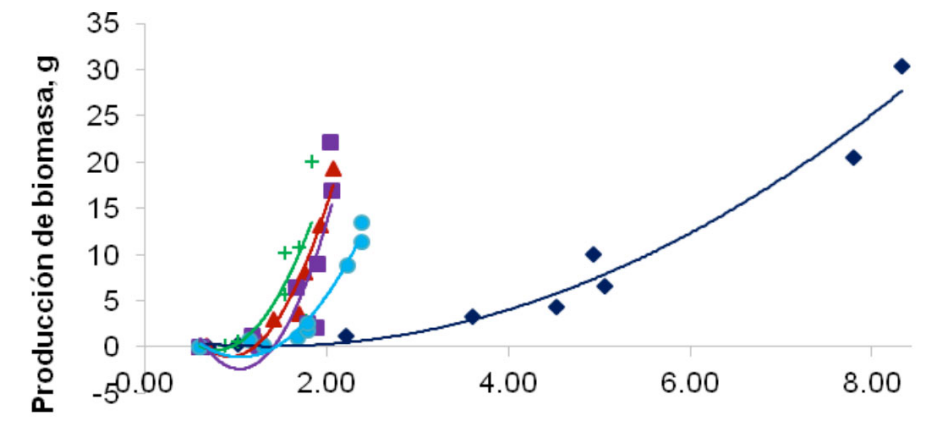

\begin{tabular}{lll} 
& \multicolumn{2}{l}{ Índice de concentración de clorofila (ICC) } \\
- $689=$ & $0,5682 x^{2}-1,5391 x+1,1049$ & $\mathrm{R}^{2}=0,96$ \\
$\Delta 592=$ & $14,386 x^{2}-26,831 x+11,464$ & $\mathrm{R}^{2}=0,94$ \\
+ Vaughn = & $12,489 x^{2}-19,538 x+7,3328$ & $\mathrm{R}^{2}=0,64$ \\
- Niner = & $17,261 x^{2}-35,894 x+16,282$ & $\mathrm{R}^{2}=0,70$ \\
- El Reno $=$ & $7,4298 x^{2}-15,628 x+7,1262$ & $\mathrm{R}^{2}=0,96$
\end{tabular}

Figura 3. Relación entre el índice de concentración de clorofila y producción de biomasa en condiciones de invernadero de cinco genotipos de pasto banderita (Bouteloua curtipendula) con potencial para su uso en revegetación de pastizales, de la semana uno a la diez después de la emergencia. Facultad de Zootecnia y Ecología (FZyE), Universidad Autónoma de Chihuahua (UACH), México. 2015.

Figure 3. Relation betweenchlorophyll concentration index and biomass production under greenhouse conditions on five sideoats grama (Bouteloua curtipendula) genotypes used in grasslands revegetation, from the first to the tenth week after the emergency. School of Animal Sciences and Ecology Autonomous University of Chihuahua (UACH), México. 2015.

obtuvo mayor PB. Sin embargo, el AE puede también estar relacionada con el cierre y la apertura de estomas. Estudios recientes señalan que estomas de menor tamaño pueden abrirse y cerrarse con mayor rapidez, lo que a su vez puede disminuir la transpiración (Drake et al., 2013; Raven, 2014; Fanourakis et al., 2015). No obstante, existe información que muestra que los estomas de la familia Poaceae poseen movimientos más rápidos que otras especies (Hetherington y Woodward, 2003), por lo que, la tasa de transpiración de los genotipos de pasto banderita con mayor AE, podría no verse afectada. Esto concuerda con lo reportado por Wilkinson (1979), quien señaló que plantas con estomas grandes son características de ambientes secos y de alta radiación solar, mientras que, las de estomas pequeños comúnmente se encuentran en zonas más húmedas y donde las plantas están expuestas a menor radiación solar. Por otro lado, el tamaño de las células, incluyendo las células guarda, se establece en parte por el tamaño del genoma de la planta (Hodgson et al., 2010). En este sentido, el genotipo E-689, por haber sido el de mayor AE, tiene una ventaja adaptativa y es más eficiente en el uso del agua. Por otro lado, en estudios recientes se ha establecido una correlación positiva entre el tamaño del genoma de las plantas con el de sus células guarda (Beaulieu et al., 2008; Hodgson et al., 2010). Por lo anterior, las diferencias en AE podrían indicar diferencias en el tamaño de los genomas de los materiales analizados en el estudio.

La clorofila es un pigmento capaz de captar fotones de luz solar de diferentes colores y convertirlos en compuestos orgánicos ricos en energía. Una manera fácil y rápida de estimar la concentración de clorofila, es mediante el cálculo del índice de concentración de clorofila (Jifon et al., 2005; Ghasemi et al., 2011). Está documentado que, la concentración de clorofila está relacionada con la producción de biomasa (Rodrigues et al., 2006; Wu et al., 2008). Esta afirmación coincide con los resultados de este estudio, donde la PB mostró correlación positiva con el ICC, y el único genotipo que presentó diferencias en el ICC fue el E-689, mismo que presentó la mayor PB. Además, el índice de concentración de clorofila está relacionado con el contenido de nitrógeno (Ghasemi et al., 2011); el cual, a su vez, está correlacionado con la concentración de proteína en plantas (Daughtry 
et al., 2000; Rincón y Ligarreto, 2010). Por lo anterior, el genotipo E-689 al presentar mayores valores en el ICC, probablemente sea el que posea mayor contenido de proteína de los genotipos estudiados.

\section{Conclusiones}

El pasto banderita puede clasificarse como una especie anfiestomática, en razón de su proporción equilibrada de estomas en las superficies adaxial y abaxial. Los genotipos de pasto banderita con menor densidad estomática y menor índice estomático, pero mayor área estomática, pueden potencialmente producir mayor cantidad de biomasa. Además, genotipos con mayor concentración de clorofila pueden ser más productivos.

Las características de los estomas y su concentración pueden ser factores determinantes en las diferencias en producción de biomasa entre genotipos de pasto banderita. Por lo anterior, las características de los estomas y el índice de concentración de clorofila, pueden establecerse como criterios para la selección de nuevos genotipos y se recomiendan para futuros programas de selección, dentro del complejo Bouteloua curtipendula.

\section{Literatura citada}

Beaulieu, J.M., I.J. Leitch, S. Patel, A. Pendharkar, and C.A. Knight. 2008. Genome size is a strong predictor of cell size and stomatal density in angiosperms. New Phytol. 179:975-986. doi:10.1111/j.1469-8137.2008.02528.x

Beltrán, S., C.A. García, J.A. Hernández, C. Loredo, J. Urrutia, L.A. González, y H.G. Gámez. 2013. "Banderilla Diana” Bouteloua curtipendula (Michx.) Torr., nueva variedad de pasto para zonas áridas y semiáridas. Rev. Mex. Cienc. PecU. 4:217-221.

Croxdale, J.L. 2000. Stomatal patterning in angiosperms. Am. J. Bot. 87:1069-1080. doi:10.2307/2656643

Daughtry, C.S.T., C.L. Walthall, M.S. Kim, E. Brown-de-Colstoun, and J.E. McMurtrey. 2000. Estimating corn leaf chlorophyll concentration from leaf and canopy reflectance. Remote Sens. Environ. 74:229-239. doi:10.1016/S0034-4257(00)00113-9

Drake, P.L., R.H. Froend, and P.J. Franks. 2013. Smaller, faster stomata: scaling of stomatal size, rate of response, and stomatal conductance. J. Exp. Bot. 64:495-505. doi:10.1093/jxb/ers347

Duke, S.O. 1994. Glandular trichomes-A focal point of chemical and structural interactions. Int. J. Plant Sci. 155:617-620.

Fanourakis, D., H. Giday, R. Milla, R. Pieruschka, K.H. Kjaer, M. Bolger, A. Vasilevski, A. Nunes-Nesi, F. Fiorani, and C.O. Ottosen. 2015. Pore size regulates operating stomatal conductance, while stomatal densities drive the partitioning of conductance between leaf sides. Ann. Bot. 115:555-565. doi:10.1093/aob/mcu247

Franks, P.J., and D.J. Beerling. 2009. Maximum leaf conductance driven by $\mathrm{CO}_{2}$ effects on stomatal size and density over geologic time. PNAS 106:10343-10347. doi:10.1073/pnas.0904209106

Franks, P.J., T.W. Doheny-Adams, Z.J. Britton-Harper, and J.E. Gray. 2015. Increasing water-use efficiency directly through genetic manipulation of stomatal density. New Phytol. 207:188-195. doi:10.1111/nph.13347

Fraser, L.H.,A. Greenall, C. Carlyle, R. Turkington, and C.R. Friedman. 2009. Adaptive phenotypic plasticity of Pseudoroegneria spicata: Response of stomatal density, leaf area and biomass to changes in water supply and increased temperature. Ann. Bot. 103:769-775. doi:10.1093/aob/men252

Ghasemi, M., K. Arzani, A. Yadollahi, S. Ghasemi, and S. Sarikhani. 2011. Estimate of leaf chlorophyll and nitrogen content in asian pear (Pyrus serotina Rehd.) by CCM-200. Not. Sci. Biol. 3:91-94. doi:10.15835/nsb315623 
Giday, H., K.H. Kjaer, D. Fanourakis, and C.O. Ottosen. 2013. Smaller stomata require less severe leaf drying to close: a case study in Rosa hydrida. J. Plant Physiol. 170:1309-1316. doi:10.1016/j.jplph.2013.04.007

Hetherington, A.H., and F.I. Woodward. 2003. The role of stomata in sensing and driving environmental change. Nature 424:901-908. doi:10.1038/nature01843

Hodgson, J.G., M. Sharafi, A. Jalili, S. Díaz, G. Montserrat-Martí, C. Palmer, B. Cerabolini, S. Pierce, B. Hamzehee, Y. Asri, Z. Jamzad, P. Wilson, J.A. Raven, S.R. Band, S. Basconcelo, A. Bogard, G. Carter, M. Charles, P. Castro-Díez, J.H. Cornelissen, G. Funes, G. Jones, M. Khoshnevis, N. Pérez-Harguindeguy, M.C. Pérez-Rontomé, F.A. Shirvany, F. Vendramini, S. Yazdani, R. Abbas-Azimi, S. Boustani, M. Dehghan, J. Guerrero-Campo, A. Hynd, E. Kowsary, F. Kazemi-Saeed, B. Siavash, P. Villar-Salvador, R. Craigie, A. Naqinezhad, A. Romo-Díez, L. de-Torres-Espuny, and E. Simmons. 2010. Stomatal vs. genome size in angiosperms: the somatic tail wagging the genome dog? Ann. Bot. 105:573584. doi:10.1093/aob/mcq011

Jifon, J.L., J.P. Syvertsen, and E. Whaley. 2005. Growth enviroment and leaf anatomy affect nondestructive estimates of chlorophyll and nitrogen in Citrus Sp. leaves. J. Amer. Soc. Hort. Sci. 130:152-158.

Klooster, B., and E. Palmer-Young. 2004. Water stress marginally increases stomatal density in E. canadensis, but not in A. gerardii. Tillers 5:35-40.

Lawson, T., and M.R. Blatt. 2014. Stomatal size, speed, and responsiveness impact on photosynthesis and water use efficiency. Plant Physiol. 164:1556-1570. doi:10.1104/pp.114.237107

Lawson, T., A.J. Simkin, G. Kelly, and D. Granot. 2014. Mesophyll photosynthesis and guard cell metabolism impacts on stomatal behaviour. New Phytol. 203:1064-1081. doi:10.1111/nph.12945

Martin, W.J., and D.P. Stimart. 2005. Stomatal density in Antirrhinum majus L.: Inheritance and trends with development. HortSci. 40:1252-1258.

Meharun-Nisa, K.Y., K.A. Siddiqui, and A.Q. Soomro. 2009. Flag leaf stomatal frequency and its interrelationship with yield and yield components in wheat (Triticum aestivum L.). Pak. J. Bot. 41:663-666.

Melgoza-Castillo, A., C. Ortega-Ochoa, C.R. Morales-Niero, P. Jurado-Guerra, C. Velez-Sanchez-Verin, M.H. Royo-Márquez, G. Quintana-Martínez, A. Lafón-Terrazas, M.T. Alarcón.Herrera, G. Bezanilla-Enríquez, y C. Pinedo-Álvarez. 2007. Propagación de plantas nativas para la recuperación de áreas degradadas: opción para mejorar ecosistemas. Tecnocienc. Chihuahua 1(3):38-41.

Morales, C.R., C. Avendaño, A. Melgoza, K. Gil, A. Quero, P. Jurado, y M. Martínez. 2016. Caracterización morfológica y molecular de poblaciones de pasto banderita (Bouteloua curtipendula) en Chihuahua, México. Rev. Mex. Cienc. Pecu. 7:455-469.

Morales-Nieto, C., A. Quero-Carillo, O. Le-Blanc, A. Hernández-Garay, J. Pérez-Pérez, y S. González-Muñoz. 2006. Caracterización de la diversidad del pasto nativo Bouteloua curtipendula (Michx) Torr. mediante marcadores de AFLP. Agrociencia 40:711-720.

Morales-Nieto, C.R., A. Quero-Carrillo, J. Pérez-Pérez, A. Hernández-Garay, y O. Le-Blanc. 2008. Caracterización morfológica de poblaciones nativas de pasto banderita [Bouteloua curtipendula (Michx.) Torr.] en México. Agrociencia 42:767-775.

Palma-Rivero, M.P., A. López-Herrera, y J.C. Molina-Moreno. 2000. Condiciones de almacenamiento y germinación de semillas de Cenchrus ciliaris L. y Andropogon gayanus Kunth. Agrociencia 34:41-48.

Parkhurst, D.F., S.C. Wong, G.D. Farquar, and I.R. Cowan. 1988. Gradients of intracellular $\mathrm{CO}_{2}$ levels across the leaf mesophyll. Plant Physiol. 86:1032-1037. doi:10.1104/pp.86.4.1032 
Perveen, A., R. Abid, and R. Fatima. 2007. Stomatal types of some dicots within flora of karachi, Pakistan. Pak. J. Bot. 39:10171023.

Raven, J.A. 2014. Speedy small stomata? J. Exp. Bot. 65:1415-1424. doi:10.1093/jxb/eru032

Reyes-López, D., J. Quiroz-Valentín, A. Kelso-Bucio, M. Huerta-Lara, C. Avendaño-Arrazate, y R. Lobato-Ortiz. 2015. Caracterización estomática de cinco especies del género Vanilla. Agron. Mesoam. 26:237-246. doi:10.15517/ am.v26i2.19279

Rincón, A., y G.A. Ligarreto. 2010. Relación entre nitrógeno foliar y el contenido de clorofila, en maíz asociado con pastos en el Piedemonte Llanero colombiano. Corpoica Cienc. Tecnol. Agropecu. 11:122-128. doi:10.21930/rcta.vol11_num2_art:202

Rivera, C., A. Zapata, G. Pinilla, J. Donato, B. Chaparro, y P. Jiménez. 2005. Comparación de la estimación de la clorofila-a mediante los métodos espectrofotométrico y fluorométrico. Acta Biol. Colomb. 10(2):95-103.

Rodrigues, L.R., T.J. Rodrigues, R. Reis, e C.V. Filho. 2006. Avaliação de características fisiológicas de cinco cultivares de Cynodon. Acta Sci. Anim. Sci. 28:245-250. doi:10.4025/actascianimsci.v28i3.36

SAS. 2006. SAS/STAT 9.1.3 User's guide. SAS Institute, Cary, NC, USA.

Schlüter, U., M. Muschak, D. Berger, and T. Altmann. 2003. Photosynthetic performance of an Arabidopsis mutant with elevated stomatal density (sdd1-1) under different light regimes. J. Exp. Bot. 54:867-874. doi:10.1093/jxb/erg087

Taylor, S.H., P.J. Franks, S.P. Hulme, E. Springgs, P.A. Christin, E.J. Edwards, F.I. Woodward, and C.P. Osborne. 2012. Photosynthetic pathway and ecological adaptation explain stomatal trait diversity amongst grasses. New Phytol. 193:387396. doi:10.1111/j.1469-8137.2011.03935.x

Walton, P.D. 1980. The production characteristics of Bromus inermis leyss and their Inheritance. Adv. Agron. 33:341-369. doi:10.1016/S0065-2113(08)60171-6

Wang, Y., X. Chen, and C.B. Xiang. 2007. Stomatal density and bio-water saving. J. Integr. Plant Biol. 49:1435-1444. doi:10.1111/j.1672-9072.2007.00554.X

Wilkinson, H.P. 1979. The plant surface (mainly leaf). In: C.R. Metalcafe, and L. Chalk, editors, Anatomy of the dicotyledons. Vol. 1. Systematic Anatomy of the Leaf and Stem. 2nd ed. Claredon Press, Oxford, GBR. p. 97-117.

Wu, C., Z. Niu, Q. Tang, and W. Huang. 2008. Estimating chlorophyll content from hyperspectral vegetation indices: modeling and validation. Agric. For. Meteorol. 148:1230-1241. doi:10.1016/j.agrformet.2008.03.005

Xu, Z., and G. Zhou. 2008. Responses of leaf stomatal density to water status and its relationship with photosynthesis in a grass. J. Exp. Bot. 59:3317-3325. doi:10.1093/jxb/ern185 